\title{
Effects of Residual Stress on the Hydro-Elastic Vibration of Circular Diaphragm
}

\author{
Junfeng Zhao, Song Yu \\ Department of Engineering Mechanics, Shandong University, Jinan, China \\ Email: zhaojunfeng@sdu.edu.cn
}

Received October 12, 2012; revised November 11, 2012; accepted November 21, 2012

\begin{abstract}
The effects of residual stress on the hydro-elastic vibration of circular diaphragm are theoretically investigated by using the added mass approach. The Kirchhoff theory of plates is used to model the elastic thin circular diaphragm on an aperture of an infinite rigid wall and in contact with a fluid on one side. The fluid is assumed to be incompressible and inviscid and the velocity potential is used to describe its irrotational motion. A non-dimensional tension parameter is defined, and the effects of the tension parameter on the frequency parameters and mode shapes of the diaphragm in the air are presented. The Hankel transform is applied to solve the fluid-diaphragm coupled system; boundary conditions are expressed by integral equations. Finally, the effects of residual stress on the non-dimensional added virtual mass incremental (NAVMI) factors of the diaphragm contact with a fluid on one side are investigated. It is found that the effects of the residual stress cannot be neglected when the edges of the circular diaphragm are clamped. The effects of residual stress for NAVMI factors can be increases $11 \%$ when the non-dimensional tension parameter is 1000 .
\end{abstract}

Keywords: Circular Diaphragm; Residual Stress; Hankel Transform; Frequency Parameter; Mode Shape; NAVMI

\section{Introduction}

Micro-machined diaphragm structures are extensively used in micro-electromechanical systems (MEMS) as biosensors in air and in liquid media [1-5]. These sensors are excited by electro-static force so that they vibrate at their resonance frequency. When a biological entity is captured by an electrode of biosensor, the resonant frequency will change. The capture mass can be measured by detection of the resonant frequency shift. On the fabrications of these multilayer micro-diaphragms, a certain amount of residual stress will be developed for a variety of reasons [6-9]. The residual stress bend the micro-diaphragm downward or upward depending on whether the residual stress is tensile or compressive. Moreover, the residual stress is an important influencing factor for the resonant frequency and sensitivity of the biosensors. Several researchers investigated the effects of residual stress on the diaphragm's resonant frequency, either theoretically or by finite element analysis [10-12]. They concluded that residual stress stiffens the diaphragm and increases its resonant frequency. But, the vibration characteristics of the diaphragm in fluid media don't studied.

The fluid structure interaction problems of plate structures partially or totally immersed in fluid have also received much attention due to their importance. Kwak [13] and Amabili et al. [14] investigated the effect of fluid on the natural frequencies of circular plates vibrating in contact with an infinite liquid surface. Amabili and Kwak [15] investigated the effect of free-surface waves on free vibrations of circular plates resting on a free surface of infinite liquid domain. Amabili et al. [16] and Liang et al. [17] gave the natural frequencies of annular plates on an aperture of an infinite rigid wall and in contact with a fluid on one side. Bauer [18] presented the coupled hydro-elastic frequencies of a liquid in a circular cylindrical rigid container, of which the free liquid surface was fully covered by a flexible membrane or an elastic circular plate. Bauer and Chiba [19] extended the study of Bauer [18] to the structure filled with incompressible viscous liquid. Amabili [20] studied the free vibrations of circular plates resting on a sloshing liquid free surface; the liquid domain was limited by a rigid cylindrical surface and a rigid flat bottom. When the biosensors are used in liquid media, the effect of the fluid on the diaphragm must be investigated [21,22]. However, the residual stress was neglected in these studies.

The objective of the present paper is to investigate the effects of residual stress on the hydro-elastic vibration of circular diaphragm. The Kirchhoff theory of plates is used to model the elastic thin circular diaphragm. The governing equation of a circular diaphragm with the residual stress is obtained in Section 2. A non-dimensional tension parameter is defined, and the effects of the ten- 
sion parameter on the frequency parameters and mode shapes of the diaphragm in the air are presented. The fluid is assumed to be incompressible and inviscid and the velocity potential is used to describe its irrotational motion. The fluid formulation of the circular diaphragm is obtained in Section 3. In Section 4, the effects of residual stress on the frequency parameters, the mode shapes and the non-dimensional added virtual mass incremental (NAVMI) factors of the diaphragm contact with a fluid on one side are investigated. Finally, Section 5 gives concluding remarks.

\section{Equation for Circular Diaphragm with Residual Stress}

Consider a thin circular diaphragm having thickness $h$, mass density $\rho_{\mathrm{D}}$, radius $a$, initial tension of the diaphragm per unit of length $T$ due to the residual stress, which is vibrating in vacuum, as sketched in Figure 1. The diaphragm is also assumed to be made of linearly elastic, homogeneous and isotropic material. The effects of shear deformation and rotary inertia are neglected. The Kirchhoff theory of plates is used to model the elastic thin circular diaphragm.

For a circular diaphragm with initial tension, the equation of motion for transverse displacement, $w$, of the diaphragm is [23]

$$
D \nabla^{4} w-T \nabla^{2} w+\rho_{\mathrm{D}} h \frac{\partial^{2} w}{\partial t^{2}}=0
$$

where $D=E h^{3} / 12\left(1-v^{2}\right)$ is the flexural rigidity of the diaphragm, $v$ is the Poisson ratio of the material, $E$ is the Young's modulus of the material. $T=\sigma_{0} h(1-v)$ is the tension force of the diaphragm per unit of length due to the residual stress, $\sigma_{0}$ is the residual stress. In addition

$$
\nabla^{2}=\frac{\partial^{2}}{\partial r^{2}}+\frac{1}{r} \frac{\partial}{\partial r}+\frac{1}{r^{2}} \frac{\partial^{2}}{\partial \theta^{2}}
$$

is the Laplace operator in the polar co-ordinates $r$ and $\theta$.

For vibration analysis of the circular diaphragm in vacuum, the transverse displacement $w$ is assumed as

$$
w(r, \theta, t)=\sum_{n=0}^{\infty} \sum_{m=0}^{\infty} W_{m n}(r, \theta) \mathrm{e}^{\mathrm{i} \omega_{m n} t}
$$

in which $m$ and $n$ are the numbers of nodal diameters and circles. Substituting Equation (3) into Equation (1), we

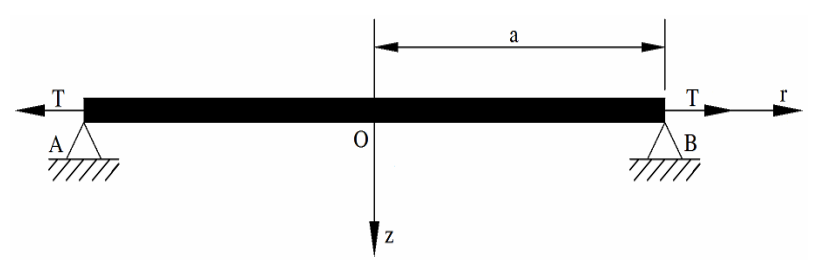

Figure 1. Schematic of a circular diaphragm with the initial tension. obtain

$$
\begin{gathered}
D \nabla^{4} W_{m n}-T \nabla^{2} W_{m n}-\rho_{\mathrm{D}} h \omega_{m n}^{2} W_{m n} \\
=\left(\nabla^{2}+\alpha_{m n}^{2}\right)\left(\nabla^{2}-\beta_{m n}^{2}\right) W_{m n}=0
\end{gathered}
$$

in which

$$
\begin{aligned}
& \alpha_{m n}^{2}=\frac{1}{2 D}\left[\sqrt{T^{2}+4 \rho_{\mathrm{D}} \omega_{m n}^{2} D}-T\right] \\
& \beta_{m n}^{2}=\frac{1}{2 D}\left[\sqrt{T^{2}+4 \rho_{\mathrm{D}} \omega_{m n}^{2} D}+T\right]
\end{aligned}
$$

where $\alpha_{m n}$ and $\beta_{m n}$ are the frequency parameters which are determined by the boundary conditions, which satisfy the following equation

$$
\left(\beta_{m n} a\right)^{2}-\left(\alpha_{m n} a\right)^{2}=\frac{T a^{2}}{D}=\zeta
$$

where $\zeta$ is a non-dimensional tension parameter which is to determined whether the diaphragm is tension dominated for the pure membrane behavior or flexural rigidity dominated for the pure plate behavior. After substituting Equation (6) into Equation (5), we obtain

$$
\begin{aligned}
& \left(\alpha_{m n} a\right)^{2}=\frac{1}{2}\left[\sqrt{\frac{4 a^{4} \rho_{\mathrm{D}} \omega_{m n}^{2}}{D}+\zeta^{2}}-\zeta\right] \\
& \left(\beta_{m n} a\right)^{2}=\frac{1}{2}\left[\sqrt{\frac{4 a^{4} \rho_{\mathrm{D}} \omega_{m n}^{2}}{D}+\zeta^{2}}+\zeta\right]
\end{aligned}
$$

The Equation (4) is satisfied when the solutions of the following equations are satisfied

$$
\left(\nabla^{2}+\alpha_{m n}^{2}\right) W_{m n}=0, \quad\left(\nabla^{2}-\beta_{m n}^{2}\right) W_{m n}=0
$$

It is possible to separate variables by substituting

$$
W_{m n}(r, \theta)=R_{m n}(r) \cos (m \theta)
$$

Substituting Equation (9) into Equation (8), then we obtain

$$
\begin{aligned}
& \frac{\mathrm{d}^{2} R_{m n}}{\mathrm{~d} r^{2}}+\frac{1}{r} \frac{\mathrm{d} R_{m n}}{\mathrm{~d} r}+\left(\alpha_{m n}^{2}-\frac{m^{2}}{r^{2}}\right) R_{m n}=0 \\
& \frac{\mathrm{d}^{2} R_{m n}}{\mathrm{~d} r^{2}}+\frac{1}{r} \frac{\mathrm{d} R_{m n}}{\mathrm{~d} r}+\left((\mathrm{i} \beta)_{m n}^{2}-\frac{m^{2}}{r^{2}}\right) R_{m n}=0
\end{aligned}
$$

Equations (10) and (11) are Bessel's equations of fractional order. The solutions can be expressed as a series form. The solution of Equation (10) is in terms of Bessel functions of the first and second kind, $J_{m}\left(\alpha_{m n} r\right)$ and $Y_{m}$ $\left(\alpha_{m n} r\right)$. The solution of Equation (11) is in terms of modified Bessel functions of the first and second kind, $I_{m}$ $\left(\beta_{m n} r\right)$ and $K_{m}\left(\beta_{m n} r\right)$.

For the general solution of circular diaphragm, the solution $R_{m n}(r)$ is obtained 


$$
\begin{aligned}
R_{m n}(r)= & A_{m n} J_{m}\left(\alpha_{m n} r\right)+B_{m n} Y_{m}\left(\alpha_{m n} r\right) \\
& +C_{m n} I_{m}\left(\beta_{m n} r\right)+D_{m n} K_{m}\left(\beta_{m n} r\right)
\end{aligned}
$$

in which $A_{m n}, B_{m n}, C_{m n}$ and $D_{m n}$ are the mode shape constants that are determined by the boundary conditions. Both $Y_{m}\left(\alpha_{m n} r\right)$ and $K_{m}\left(\beta_{m n} r\right)$ are singular at $r=0$. Thus, for a diaphragm with no central hole, we set $B_{m n}=D_{m n}=$ 0 . The Equation (12) becomes

$$
R_{m n}(r)=A_{m n} J_{m}\left(\alpha_{m n} r\right)+C_{m n} I_{m}\left(\beta_{m n} r\right)
$$

Substituting Equation (13) into Equation (9), we obtain the mode shape as follows

$$
W_{m n}(r, \theta)=\left[A_{m n} J_{m}\left(\alpha_{m n} r\right)+C_{m n} I_{m}\left(\beta_{m n} r\right)\right] \cos (m \theta)
$$

To simplify the computation, the mode shape constants $A_{m n}$ and $C_{m n}$ are normalized so that

$$
\int_{0}^{1} R_{m n}^{2}(\rho) \rho \mathrm{d} \rho=1
$$

where $\rho=r / a$ is the ratio between the radius $r$ and the maximum radius $a$.

\section{Fluid Formulation}

We consider the free vibrations of the circular diaphragm placed in an aperture of an infinite rigid wall and in contact with a fluid on one side. The mode shapes of the diaphragm vibrating in constant with a fluid are assumed to be equal to those of the diaphragm vibrating in a vacuum. This hypothesis was also used to study vibrations of circular plates in contact with fluids [13-17]. In fact, although natural frequencies are considerably reduced by the presence of a fluid, mode shapes undergo only small changes. We study the velocity potential of an incompressible and inviscid fluid in contact with a circular diaphragm on one side. The fluid movement, considered only due to the diaphragm's vibration, is assumed to be irrotational. This movement can be described by the velocity potential

$$
\Phi(r, \theta, z, t)=\operatorname{Re}\left\{\phi(r, \theta, z) \mathrm{i} \bar{\omega}_{m n} \mathrm{e}^{\mathrm{i} \bar{\omega}_{m n} t}\right\}
$$

where $\phi$ is the spatial distribution of the velocity potential, $\bar{\omega}_{m n}$ is the circular frequency of the diaphragm in contact with fluid. As a consequence of the hypotheses, $\phi$ $(r, \theta, z)$ satisfies the Laplace equation

$$
\nabla^{2} \phi=\frac{\partial^{2} \phi}{\partial r^{2}}+\frac{1}{r} \frac{\partial \phi}{\partial r}+\frac{1}{r^{2}} \frac{\partial^{2} \phi}{\partial \theta^{2}}+\frac{\partial^{2} \phi}{\partial z^{2}}=0 \quad \text { in } F
$$

where $F$ represents the unbounded fluid domain. The fluid is in contact with the diaphragm through the surface denoted by $S_{1}$ and in contact with an infinite rigid wall through the surface denoted by $S_{2}$, as sketches Figure 2 . Due to the fluid domain is unbounded, the surface $S_{\infty}$ exists at infinity.

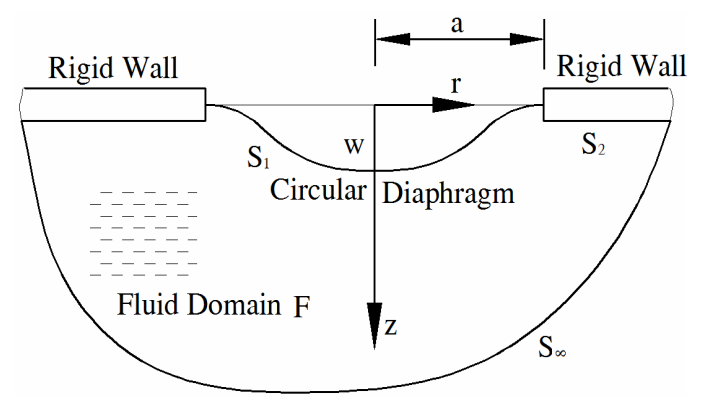

Figure 2. The circular diaphragm and the fluid domain.

As assumed in the hypotheses, the diaphragm mode shapes in a vacuum and in fluids are unchanged, therefore, the displacement $w$ obtained from Equation (3) is imposed at the fluid-plate interface. The contact between the fluid and diaphragm is assured by the equation $[13,14]$

$$
v_{z}=-\frac{\partial \phi}{\partial z}=-R_{m n}(r) \cos (m \theta) \quad \text { on } S_{1}
$$

The condition of an impermeable rigid wall on $S_{2}$ is

$$
\frac{\partial \phi}{\partial z}=0 \quad \text { on } S_{2}
$$

Equations (18) and (19) give a Neumann problem. Moreover, there is the radiation condition that the spatial velocity potential $\phi$ and the velocity of the fluid go to zero when the distance from the diaphragm becomes very large $[13,14]$

$$
\begin{aligned}
& \phi(r, \theta, z), \frac{\partial \phi(r, \theta, z)}{\partial r}, \frac{\partial \phi(r, \theta, z)}{\partial z} \rightarrow 0 \\
& \text { for } r, z \rightarrow \infty \text { on } S_{\infty}
\end{aligned}
$$

Equation (17) can be simplified by separation of variables

$$
\phi(r, \theta, z)=\Psi(r, z) \cos (m \theta)
$$

where $\Psi$ is the solution of

$$
\frac{\partial^{2} \Psi}{\partial r^{2}}+\frac{1}{r} \frac{\partial \Psi}{\partial r}+\frac{\partial^{2} \Psi}{\partial z^{2}}-\frac{m^{2}}{r^{2}} \Psi=0 \quad \text { in } F
$$

The boundary conditions for $\Psi$ are directly obtained from Equations (18)-(20) as

$$
\begin{gathered}
\left.\frac{\partial \Psi(r, z)}{\partial z}\right|_{z=0}=R_{m n}(r) \quad \text { on } S_{1} \\
\left.\frac{\partial \Psi}{\partial z}\right|_{z=0}=0 \quad \text { on } S_{2} \\
\Psi(r, z), \frac{\partial \Psi(r, z)}{\partial r}, \frac{\partial \Psi(r, z)}{\partial z} \rightarrow 0 \\
\text { for } r, z \rightarrow \infty \quad \text { on } S_{\infty}
\end{gathered}
$$

Using the Hankel transformation [24], we obtain the 
relation

$$
\bar{\Psi}(\xi, z)=\int_{0}^{\infty} r \Psi(r, z) J_{m}(\xi r) \mathrm{d} r
$$

By integrating by parts, we can derive the relation

$$
\begin{aligned}
& \int_{0}^{\infty} r\left(\frac{\partial^{2} \Psi}{\partial r^{2}}+\frac{1}{r} \frac{\partial \Psi}{\partial r}-\frac{m^{2}}{r^{2}} \Psi\right) J_{m}(\xi r) \mathrm{d} r \\
& =-\xi^{2} \int_{0}^{\infty} r \Psi(r, z) J_{m}(\xi r) \mathrm{d} r=-\xi^{2} \Psi(\xi, z)
\end{aligned}
$$

At the same time, we have for the third term of Equation (22)

$$
\int_{0}^{\infty} r \frac{\partial^{2} \Psi}{\partial z^{2}} J_{m}(\xi r) \mathrm{d} r=\frac{\mathrm{d}^{2}}{\mathrm{~d} z^{2}} \bar{\Psi}(\xi, z)
$$

Using Equations (27) and (28) reduces the partial differential Equation (22) to the ordinary differential equation

$$
\frac{\mathrm{d}^{2}}{\mathrm{~d} z^{2}} \bar{\Psi}(\xi, z)-\xi^{2} \bar{\Psi}(\xi, z)=0
$$

Boundary condition Equation (25) requires that the solution of Equation (29) consists of the attenuating part only

$$
\bar{\Psi}(\xi, z)=B(a \xi) \mathrm{e}^{-\xi z} \quad z \geq 0
$$

where $B(a \xi)$ is a function that must be determined. From the inversion formula for the Hankel transformation along with Equation (30), we obtain

$$
\begin{aligned}
\Psi(r, z) & =\int_{0}^{\infty} \xi \bar{\Psi}(\xi, z) J_{m}(\xi r) \mathrm{d} \xi \\
& =\int_{0}^{\infty} \xi B(a \xi) \mathrm{e}^{-\xi z} J_{m}(\xi r) \mathrm{d} \xi
\end{aligned}
$$

Inserting Equation (31) into the boundary condition Equations (23) and (24) give

$$
\begin{gathered}
\int_{0}^{\infty} \xi^{2} B(a \xi) J_{m}(\xi r) \mathrm{d} \xi=-R_{m n}(r), \quad 0 \leq r \leq a \\
\int_{0}^{\infty} \xi^{2} B(a \xi) J_{m}(\xi r) \mathrm{d} \xi=0, \quad r>a
\end{gathered}
$$

Introducing the non-dimensional variables as

$$
\rho=\frac{r}{a}, \quad \eta=a \xi, \quad A(\eta)=\eta B(\eta)
$$

The integral Equations (32) and (33) can be written in the following standard form

$$
\begin{gathered}
\int_{0}^{\infty} \eta A(\eta) J_{m}(\eta \rho) \mathrm{d} \eta=-a^{3} R_{m n}(\rho), \quad 0 \leq \rho \leq 1 \\
\int_{0}^{\infty} \eta A(\eta) J_{m}(\eta \rho) \mathrm{d} \eta=0, \quad \rho>1
\end{gathered}
$$

where

$$
R_{m n}(\rho)=A_{m n} J_{m}\left(\alpha_{m n} a \rho\right)+C_{m n} I_{m}\left(\beta_{m n} a \rho\right)
$$

The solution of the integral Equations (35) and (36), can be obtained by using the properties of the Hankel transform [24]. In fact, since the Equation (36) is equal to zero, we obtain

$$
A(\eta)=-a^{3} \int_{0}^{1} \rho R_{m n}(\rho) J_{m}(\eta \rho) \mathrm{d} \rho=-a^{3} H_{m n}(\eta)
$$

in which [25]

$$
\begin{aligned}
& H_{m n}(\eta)=A_{m n} H_{A m n}(\eta)+C_{m n} H_{C m n}(\eta) \\
& H_{A m n}(\eta) \\
&= \frac{\alpha_{m n} a J_{m+1}\left(\alpha_{m n} a\right) J_{m}(\eta)-\eta J_{m}\left(\alpha_{m n} a\right) J_{m+1}(\eta)}{\alpha_{m n}^{2} a^{2}-\eta^{2}} \\
&= \frac{H_{C m n}(\eta)}{\beta_{m n} a I_{m+1}\left(\beta_{m n} a\right) J_{m}(\eta)+\eta I_{m}\left(\beta_{m n} a\right) J_{m+1}(\eta)} \\
& \beta_{m n}^{2} a^{2}+\eta^{2}
\end{aligned}
$$

Inserting Equation (38) into Equation (31) and using Equation (34), we obtain the function $\Psi$ at the fluid-plate interface

$$
\Psi(\rho, 0)=-a \int_{0}^{\infty} H_{m n}(\eta) J_{m}(\eta \rho) \mathrm{d} \eta
$$

Using the Rayleigh quotient for the coupled vibrations, the squares of the natural frequencies of the diaphragm in a vacuum are proportional to the ratio between the maximum potential energy of the diaphragm $V_{D}$ and its kinetic energy $T_{D}$ [26]. Due to the mode shapes of the diaphragm vibration in constant with a fluid are assumed to be equal to those of the diaphragm vibration in a vacuum, the squares of the natural frequencies in fluid are proportional to the ratio between the maximum potential energy of the diaphragm $V_{D}$ and the sum of the kinetic energies of both the diaphragm $T_{D}$ and the fluid $T_{F}$. Therefore, we can obtain

$$
\begin{gathered}
f_{V}^{2} \propto\left(\frac{V_{D}}{T_{D}}\right)_{\text {vacuum }} \\
f_{F}^{2} \propto\left(\frac{V_{D}}{T_{D}+T_{F}}\right)_{\text {fluid }}
\end{gathered}
$$

Since the mode shapes in a vacuum and in fluids are assumed to be the same, the maximum potential energies $V_{D}$ and the kinetic energy $T_{D}$ are not changed when evaluated in vacuum or in fluids. By using Equations (43) and (44), the following relation between natural frequencies in a vacuum $f_{V}$ and natural frequencies in fluids $f_{F}$ is obtained

$$
f_{F}=\frac{f_{V}}{\sqrt{1+\beta_{m n}}}
$$

where $\beta_{m n}$ is the added virtual mass incremental (AVMI) factor $[13,14]$. This factor is given by the ratio between the kinetic energy of the fluids and the kinetic energy of the diaphragm 


$$
\beta_{m n}=\frac{T_{F}}{T_{D}}
$$

The AVMI factor can be made dimensionless

$$
\beta_{m n}=\Gamma_{m n} \frac{\rho_{F} a}{\rho_{D} h}
$$

where $\rho_{F}$ and $\rho_{D}$ are the fluid's and diaphragm's mass density, respectively, and $\Gamma_{m n}$ is the non-dimensionalized added virtual mass incremental (NAVMI) factor [15-17]. Therefore, natural frequencies of a circular diaphragm vibrating in contact with a fluid are related to the modal properties of vibration in a vacuum.

By using the hypothesis of irrotational movement of the fluid and simple connection of the fluid domain, the kinetic energy of the fluid can be evaluated from its boundary motion [13-17]. In fact, as a consequence of Green's Theorem applied to the harmonic function $\phi$, we obtain

$$
T_{F}=-\frac{\rho_{F}}{2} \int_{0}^{2 \pi} \int_{0}^{\infty} \frac{\partial \Psi(r, 0)}{\partial z} \Psi(r, 0) \cos ^{2}(m \theta) r \mathrm{~d} r \mathrm{~d} \theta
$$

The function $\partial \psi / \partial z$ is always zero on the boundary of the fluid domain, except for the surface in contact with the plate, therefore Equation (48) reduces to

$$
\begin{aligned}
T_{F} & =-\frac{\rho_{F}}{2} \psi_{\theta} \int_{0}^{a} r R_{m n}(r) \Psi(r, 0) \mathrm{d} r \\
& =-\frac{\rho_{F}}{2} a^{2} \psi_{\theta} \int_{0}^{1} \rho R_{m n}(\rho) \Psi(\rho, 0) \mathrm{d} \rho
\end{aligned}
$$

where $\psi_{\theta}=2 \pi$ for $m=0, \psi_{\theta}=\pi$ for $m>0$. Upon substituting Equation (42) into Equation (49) and using Equation (38), the result is found to be

$$
T_{F}=\frac{\rho_{F}}{2} a^{3} \psi_{\theta} \int_{0}^{\infty} H_{m n}^{2}(\eta) \mathrm{d} \eta
$$

The kinetic energy of the circular diaphragm is

$$
T_{D}=\frac{\rho_{D}}{2} h \int_{0}^{2 \pi} \int_{0}^{a} R_{m n}^{2}(r) \cos ^{2}(m \theta) r \mathrm{~d} r \mathrm{~d} \theta
$$

Integration with respect to $\theta$, and using the normalization criterion Equation (15), then we obtain

$$
\begin{aligned}
T_{D} & =\frac{\rho_{D}}{2} h \psi_{\theta} \int_{0}^{a} R_{m n}^{2}(r) r \mathrm{~d} r \\
& =\frac{\rho_{D}}{2} h a^{2} \psi_{\theta} \int_{0}^{1} R_{m n}^{2}(\rho) \rho \mathrm{d} \rho=\frac{\rho_{D}}{2} h a^{2} \psi_{\theta}
\end{aligned}
$$

By using Equations (46), (47), (50) and (52), the NAVMI factors are found to be given by

$$
\Gamma_{m n}=\frac{T_{F} \rho_{D} h}{T_{D} \rho_{F} a}=\int_{0}^{\infty} H_{m n}^{2}(\eta) \mathrm{d} \eta
$$

This quantity must be evaluated numerically, because the integral cannot be expressed in terms of elementary functions. However, upon inserting the modal parameters $\alpha_{m n}, \beta_{m n}, A_{m n}$ and $C_{m n}$, the NAVMI factors can be quickly computed.

\section{Numerical Results and Discussion}

\subsection{Numerical Results}

To illustrate the effects of the residual stress, the frequency parameters, $\alpha_{m n} a$, the mode shapes and the nondimensional added virtual mass incremental (NAVMI) factors are been studied for the circular diaphragm with the clamped edge. For the circular diaphragm with clamped edge, the boundary conditions are

$$
W_{m n}=0, \quad \frac{\partial W_{m n}}{\partial r}=0 \quad \text { on } r=a
$$

Using Equation (14) and Equation (54), we obtain

$$
\left.\begin{array}{l}
A_{m n} J_{m}\left(\alpha_{m n} a\right)+C_{m n} I_{m}\left(\beta_{m n} a\right)=0 \\
-A_{m n} \alpha_{m n} J_{m+1}\left(\alpha_{m n} a\right)+C_{m n} \beta_{m n} I_{m+1}\left(\beta_{m n} a\right)=0
\end{array}\right\}
$$

Due to Equation (55) has non-zero solution, the coefficient determinant of Equation (55) must be zero, which gives

$$
\left|\begin{array}{cc}
J_{m}\left(\alpha_{m n} a\right) & I_{m}\left(\beta_{m n} a\right) \\
-\alpha_{m n} J_{m+1}\left(\alpha_{m n} a\right) & \beta_{m n} I_{m+1}\left(\beta_{m n} a\right)
\end{array}\right|=0
$$

The characteristic equation of the circular diaphragm with clamped edge can be obtained

$$
\begin{aligned}
& \alpha_{m n} a J_{m+1}\left(\alpha_{m n} a\right) I_{m}\left(\beta_{m n} a\right) \\
& +\beta_{m n} a I_{m+1}\left(\beta_{m n} a\right) J_{m}\left(\alpha_{m n} a\right)=0
\end{aligned}
$$

Using Equations (6) and Equation (57), the accurate frequency parameters, $\alpha_{m n} a$, of the circular diaphragm with clamped edge can be determined.

To find the mode shapes, we formulate from Equation (55)

$$
C_{m n}=-A_{m n} \frac{J_{m}\left(\alpha_{m n} a\right)}{I_{m}\left(\beta_{m n} a\right)}
$$

This gives the mode shapes expression

$W_{m n}=A_{m n}\left[J_{m}\left(\alpha_{m n} r\right)-\frac{J_{m}\left(\alpha_{m n} a\right)}{I_{m}\left(\beta_{m n} a\right)} I_{m}\left(\beta_{m n} r\right)\right] \cos (m \theta)(59)$

Setting Equation (59) equals to zero defines the node lines. It turns out that there will be concentric circles and diametrical lines. The number of concentric circles will be $n$ and the number of diametrical lines will be $m$.

For illustration purpose, the circular diaphragm considered here is taken to be made of silicon with the following material properties: $E=170 \mathrm{GPa}, v=0.3$. The frequency parameters, $\left(\alpha_{m n} a\right)$, are available for circular plates which were given by Vogel and Skinner [27] and 
for circular membranes which were given by E. C. Wente [28]. We have computed the frequency parameters and mode shapes in order to provide more accurate results. The frequency parameters can be obtained by the Equation (57) and the mode shapes can be obtained by Equation (59). The roots of these equations were found by using the software Fortran95.

\subsection{Discussion of Results}

In Table 1, the frequency parameters, $\left(\alpha_{m n} a\right)$, for the circular diaphragm with the clamped edge are reported for the different non-dimensional tension parameter $(\zeta)$. When the non-dimensional tension parameter is zero, the diaphragm model is reduced to the plate [27]. It is found the results are the same with the circular plates when the tension parameter is zero. When the non-dimensional tension parameter is infinite, the diaphragm model is reduced to the membrane [28]. The frequency parameters are the same with the circular membranes. It indicates that the present model is valid for the diaphragm with the clamped edge.

In Figure 3, the frequency parameters of the four different models for the circular diaphragms with clamped edges are investigated. It is clearly seen that the frequencies parameters decreases rapidly with the increasing value of the non-dimensional tension parameter. When the tension parameter is very small, the frequency parameters close to the results of the plates.

Table 1. Comparison of frequency parameters, $\left(\alpha_{m n} a\right)$, for circular diaphragms with clamped edge, $v=0.3$.

\begin{tabular}{|c|c|c|c|c|c|c|}
\hline \multirow{3}{*}{$m$} & \multirow{3}{*}{$n$} & \multirow{3}{*}{$\begin{array}{c}\text { Plates } \\
\text { Vogel et al. } \\
{[27]}\end{array}$} & \multicolumn{3}{|c|}{ Present work } & \multirow{3}{*}{$\begin{array}{c}\text { Membrane } \\
\text { E. C.Wente [28] }\end{array}$} \\
\hline & & & \multirow{2}{*}{$\begin{array}{c}\text { plates } \\
\zeta=0\end{array}$} & \multicolumn{2}{|c|}{ diaphragm } & \\
\hline & & & & $\zeta=10^{2}$ & $\zeta=10^{4}$ & \\
\hline 0 & 0 & 3.19622 & 3.19622 & 2.65624 & 2.42910 & 2.40483 \\
\hline 0 & 1 & 6.30644 & 6.30644 & 5.99518 & 5.57569 & 5.52008 \\
\hline 1 & 0 & 4.61090 & 4.61090 & 4.20075 & 3.87036 & 3.83171 \\
\hline 1 & 1 & 7.79927 & 7.79927 & 7.55770 & 7.08615 & 7.01559 \\
\hline
\end{tabular}

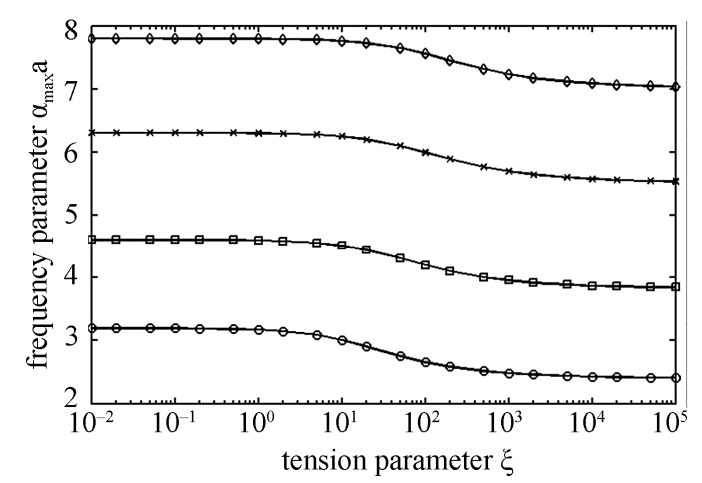

Figure 3. Frequency parameters of the clamped circular diaphragm as function of the tension parameter. $m, n$ values: $-0-, 0,0 ;-\square-, 1,0 ;-\times-, 0,1 ;-\diamond-, 1,1$.
When the tension parameter is very larger, the frequency parameters close to the results of the membranes. Therefore, the vibration of a diaphragm structure depends on whether the structure behaves as a tension dominated membrane or a flexural rigidity dominated plate.

In Figure 4, the first mode shapes of the circular diaphragm with clamped edges for the different non-dimensional tension parameters are investigated. Mode shapes with zero nodal diameters $(m=0)$, zero nodal circles $(n=0)$ and $v=0.3$ are assumed. It is clearly observed from Figure 4 that the normalized maximum amplitude of the first mode shape by the present model is about 3.31 when the non-dimensional tension parameter is equal to 0 . However, the normalized maximum amplitude of the first mode shape by the present model is about 2.75 when the non-dimensional tension parameter is equal to 10,000 . The normalized maximum amplitude by the present model when the non-dimensional tension parameter is equal to 10,000 decreases approximately 17 percent than that predicted by the plate model.

In Figure 5, the second mode shapes of the circular diaphragms with clamped edges for the different nondimensional tension parameters are investigated. It is clearly observed from Figure 5 that the normalized

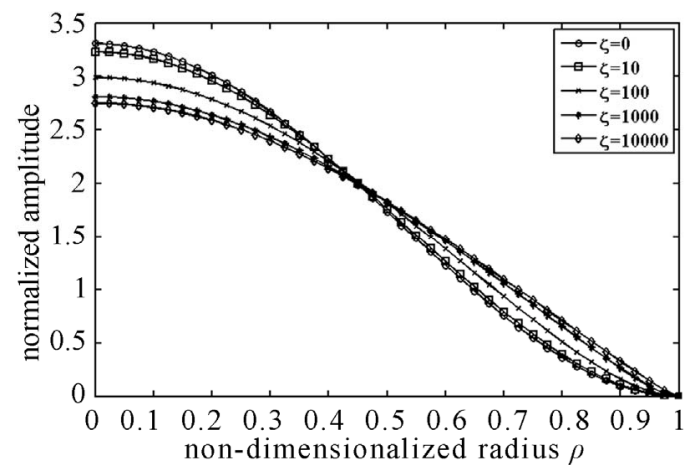

Figure 4. Mode shapes comparison of circular diaphragm for different tension parameter.

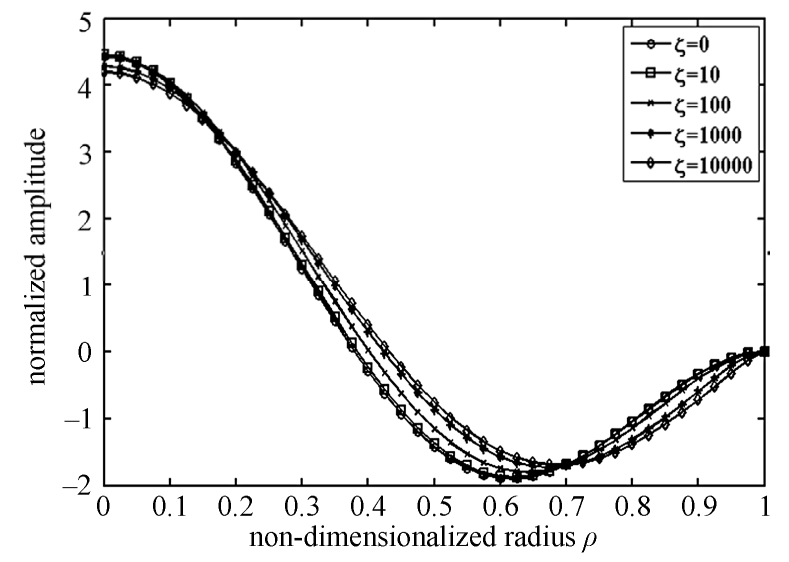

Figure 5. A comparison of mode shapes of circular diaphragm for different tension parameters. 
maximum amplitude of the second mode shape by the present model is about 4.43 when the non-dimensional tension parameter is equal to 0 . However, the normalized maximum amplitude of the second mode shape by the present model is about 4.20 when the non-dimensional tension parameter is equal to 10000 . The normalized maximum amplitude by the present model when the nondimensional tension parameter is equal to 10000 decreases approximately 5.2 percent than that predicted by the plate model. Therefore, it is insufficient that the frequency parameters and the mode shapes of the circular diaphragm with the clamped edges are predicted when the tension due to the residual stress is neglected.

The NAVMI factors, $\Gamma_{m n}$, are obtained by numerical integration of Equation (53). These factors, for clamped circular diaphragms having the non-dimensional tension parameters $\zeta$ equal to $0,10,100,1000$ and 10000 , are given in Table 2 with six frequency parameters for $m$ and $n$. When the non-dimensional tension parameter goes to zero, the diaphragm with the clamped edge becomes a clamped plate. Table 2 is shown to give a comparison between numerical results obtained by the present model and the results of the Amabili [16] for circular plates. Differences are always less than $1 \%$; the error can be attributed to the accuracy of the calculation. It indicates that the present model is valid. Therefore, for the different non-dimensional tension parameters, the NAVMI factors, $\Gamma_{m n}$, are different. It is clearly observed from Table 2 that the NAVMI factors are increase for the frequency parameter $n=0$, however, the NAVNI factors are decrease for the frequency parameter $n>0$ when the non-dimensional tension parameters is smaller than 1000 . The NAVMI factors of Table 2 are presented in Figure $\mathbf{6}$ in a graph as a function of the non-dimensional tension parameter.

It is clearly observed from Figure 6 that not all modes have a monotonic behavior with the non-dimensional tension parameter; moreover, the fundamental mode, $(m$ $=0, n=0$ ), shows the largest change with the non-dimensional tension parameter. The NAVMI factor for the fundamental mode increases approximately $11 \%$ when the non-dimensional tension parameter is equal to 1000 .

Table 2. Comparison of NAVMI factors, $\Gamma_{m n}$, for circular diaphragms with clamped edge, $v=0.3$.

\begin{tabular}{ccccccc}
\hline & & \multirow{2}{*}{$\begin{array}{c}\text { Amabili [18] } \\
m\end{array}$} & $n$ & plates & plates & \multicolumn{3}{c}{ diaphragms } \\
\cline { 4 - 7 } & & & $\zeta=0$ & $\zeta=10^{0}$ & $\zeta=10^{2}$ & $\zeta=10^{4}$ \\
\hline 0 & 0 & 0.65381 & 0.65394 & 0.65493 & 0.69337 & 0.73936 \\
0 & 1 & 0.27613 & 0.27617 & 0.27553 & 0.25751 & 0.25934 \\
0 & 2 & 0.16513 & 0.16515 & 0.16496 & 0.15578 & 0.15097 \\
1 & 0 & 0.29883 & 0.29883 & 0.29903 & 0.30914 & 0.32696 \\
1 & 1 & 0.16914 & 0.16913 & 0.16903 & 0.16566 & 0.16872 \\
1 & 2 & 0.11591 & 0.11591 & 0.11586 & 0.11337 & 0.11271 \\
\hline
\end{tabular}

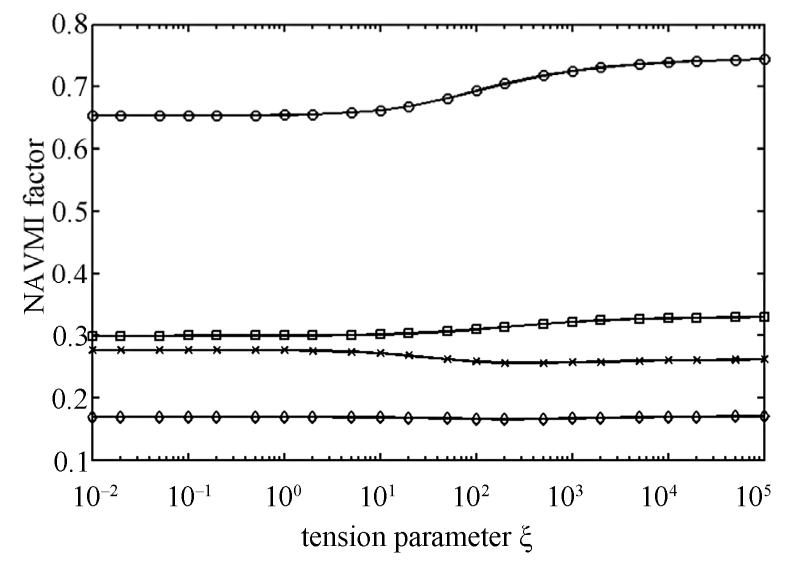

Figure 6. NAVMI factors of the clamped circular diaphragm as function of the tension parameter. $m, n$ values: $-0-, 0,0 ;-\square-, 1,0 ;-\times-, 0,1 ;-\diamond-, 1,1$.

However, the NAVMI factor for the mode with $m=0$ and $n=1$ decrease approximately $7.3 \%$ when the nondimensional tension parameter is equal to 1000 . Therefore, the residual stress must be considered when we calculate the NAVMI factors of the clamped circular diaphragms.

\section{Conclusion}

The effects of residual stress on the hydro-elastic vibration of circular diaphragm are theoretically investigated by using the added mass approach. The Kirchhoff theory of plates is used to model the elastic thin circular diaphragm on an aperture of an infinite rigid wall and in contact with a fluid on one side. The fluid is assumed to be incompressible and inviscid and the velocity potential is be used to describe its irrotational motion. It is assumed that the mode shapes are not changed by the fluid. The Hankel transform is applied to solve the fluid-diaphragm coupled system; boundary conditions are expressed by integral equations. Finally, the effects of residual stress on the non-dimensional added virtual mass incremental (NAVMI) factors of the diaphragm contact with a fluid on one side are investigated. It is found that the effects of the residual stress cannot be neglected when the boundary conditions of the circular diaphragm is clamped. The effects of residual stress for NAVMI factors can be increased $11 \%$ when the non-dimensional tension parameter is 1000 . The importance of these factors is that they are dimensionless, so that they can be applied to circular diaphragm with different dimensions and material, therefore the numerical data given in present paper can be quickly used for the design of micro-diaphragm in MEMS.

\section{REFERENCES}

[1] P. Muralt, "Ferroelectric Thin Films for Microsensors and 
Actuators: A Review," Journal of Micromechanics and Microengineering, Vol. 10, No. 2, 2000, pp. 136-146. doi:10.1088/0960-1317/10/2/307

[2] Y. Xin, Z. Li, L. V. Odum, Z. Y. Cheng and Z. Xu, "Piezoelectric Diaphragm as a High Performance Biosensor Platform," Applied Physics Letters, Vol. 89, No. 22, 2006, Article ID: 223508. doi:10.1063/1.2396919

[3] A. K. Isamil, J. S. Burdess, A. J. Harris, C. J. McNeil, J. Hedley, et al., "The Principle of a MEMS Circular Diaphragm Mass Sensor," Journal Micromechanics Microengineering, Vol. 16, No. 8, 2006, pp. 1487-1493. doi:10.1088/0960-1317/16/8/008

[4] A. K. Isamil, J. S. Burdess, A. J. Harris, G. Suarez, N. Keegan, et al., "The Fabrication, Characterization and Testing of a MEMS Circular Diaphragm Mass Sensor," Journal Micromechanics Microengineering, Vol. 18, No. 2, 2006, Article ID: 25021. doi:10.1088/0960-1317/18/2/025021

[5] M. Olfatnia, T. Xu, J. M. Miao, L. S. Ong, X. M. Jing, et al., "Piezoelectric Circular Microdiaphragm Based Pressure Sensors," Sensors and Actuators A: Physical, Vol. 163, No. 1, 2010, pp. 32-36.

doi:10.1016/j.sna.2010.06.016

[6] W. D. Nix, "Mechanical Properties of Thin Films," Metallurgical and Materials Transactions A, Vol. 20, No. 11, 1989, pp. 2217-2240.

[7] C. V. Thompson and R. Carel, "Stress and Grain Growth in Thin Films," Journal of the Mechanics and Physics of Solids, Vol. 44, No. 5, 1996, pp. 657-673. doi:10.1016/0022-5096(96)00022-1

[8] E. Hong, R. Smith, S. V. Krishnaswamy, C. B. Freidholff, and S. Trolier-McKinstry, "Residual Stress Development in $\mathrm{Pb}(\mathrm{Zr}, \mathrm{Ti}) \mathrm{O}_{3} / \mathrm{ZrO}_{2} / \mathrm{SiO}_{2}$ Stacks for Piezoelectric Microactuators," Thin Solid Films, Vol. 510, No. 1-2, 2006, pp. 213-221. doi:10.1016/j.tsf.2005.12.300

[9] M. Olfatnia, T. Xu, L. S. Ong, J. M. Miao and Z. H. Wang, "Investigation of Residual Stress and its Effects on the Vibrational Characteristics of Piezoelectric Based Multilayered Microdiaphragms," Journal of Micromechanics and Microengineering, Vol. 20, No. 1, 2010, Article ID: 15007. doi:10.1088/0960-1317/20/1/015007

[10] S. Lee, T. Tanaka, K. Inoue, J. M. Kim, Y. E. Shin, et al., "Stress Influences on the Ultrasonic Transducers," Sensors Actuators A, Vol. 119, No. 2, 2005, pp. 405-411. doi:10.1016/j.sna.2004.10.026

[11] P. Muralt, A. Kholkin, M. Kohli and T. Maeder, "Piezoelectric Actuation of PZT Thin Film Diaphragms at Static and Resonant Conditions," Sensors Actuators A, Vol. 53, No. 1-3, 1996, pp. 398-404. doi:10.1016/0924-4247(96)01139-9

[12] L. F. Ge, "Dynamic Behavior of Micro-diaphragms and Its Characterized Description," Science China Series G, Vol. 52, No. 9, 2009, pp. 1345-1356.

[13] M. K. Kwak, "Hydroelastic Vibration of Circular Plates," Journal of Sound and Vibration, Vol. 201, No. 3, 1997, pp. 293-303. doi:10.1006/jsvi.1996.0775

[14] M. Amabili, G. Dalpiaz and C. Santolini, "Free Edge Circular Plates Vibrating in Water," Modal Analysis: The
International Journal of Analytical and Experimental Modal Analysis, Vol. 10, No. 1, 1995, pp. 187-202.

[15] M. Amabili and M. K. Kwak, "Vibration of Circular Plates on a Free Fluid Surface: Effect of Surface Waves," Journal of Sound and Vibration, Vol. 226, No. 3, 1999, pp. 407-424. doi:10.1006/jsvi.1998.2304

[16] M. Amabili, G. Frosali and M. K. Kwak, "Free Vibrations of Annular Plates Coupled with Fluids," Journal of Sound and Vibration, Vol. 191, No. 5, 1996, pp. 825-846. doi:10.1006/jsvi.1996.0158

[17] C. C. Liang, Y. S. Tai and P. L. Li, "Natural Frequencies of Annular Plates Having Contact with Fluid," Journal of Sound and Vibration, Vol. 228, No. 5, 1999, pp. 11671181. doi:10.1006/jsvi.1999.2463

[18] H. F. Bauer, "Coupled Frequencies of a Liquid in a Circular Cylindrical Container with Elastic Liquid Surface Cover," Journal of Sound and Vibration, Vol. 180, No. 5, 1995, pp. 689-704. doi:10.1006/jsvi.1995.0109

[19] H. F. Bauer and M. Chiba, "Hydroelastic Viscous Oscillations in a Circular Cylindrical Container with an Elastic Cover," Journal of Fluids Structures, Vol. 14, No. 6, 2000, pp. 917-936. doi:10.1006/jfls.2000.0296

[20] M. Amabili, "Vibrations of Circular Plates Resting on a Sloshing Liquid: Solution of the Fully Coupled Problem," Journal of Sound and Vibration, Vol. 245, No. 2, 2001, pp. 261-283. doi:10.1006/jsvi.2000.3560

[21] W. P. Mason, "Electromechanical Transducers and Wave Filters," 2nd Edition, Van Bistrabd D. Company Inc., New York, 1948.

[22] A. Park, F. R. Blom, M. Elwenspoek and S. J. Lammerink, "Q-Factor and Frequency Shift of Resonating Silicon Diaphragms in Air," Sensors and Actuators A, Vol. 27, No. 1-3, 1991, pp. 691-698. doi:10.1016/0924-4247(91)87072-B

[23] M. Olfatnia, Z. Shen, J. M. Miao, L. S. Ong, T. Xu, et al., "Medium Damping Influences on the Resonant Frequency and Quality Factor of Piezoelectric Circular Microdiaphragm Sensors," Journal Micromechanics Microengineering, Vol. 21, No. 4, 2011, Article ID: 45002. doi:10.1088/0960-1317/21/4/045002

[24] I. N. Sneddon, "Mixed Boundary Value Problems in Potential Theory," North-Holland Pub. Co., Amsterdam, 1966.

[25] A. P. Prudnikov, Yu. A. Brychkov and O. I. Marichev, "Integrals and Series. Special Functions," Gordon and Breach, New York, 1986.

[26] F. Zhu, "Rayleigh Quotients for Coupled Free Vibrations," Journal of Sound and Vibration, Vol. 171, No. 5, 1994, pp. 641-649. doi:10.1006/jsvi.1994.1146

[27] S. M. Vogel and D. W. Skinner, "Natural Frequencies of Transversely Vibrating Uniform Annular Plates," Journal of Applied Mechanics, Vol. 32, No. 4, 1965, pp. 926-931. doi:10.1115/1.3627337

[28] E. C. Wente, "A Condenser Transmitter as a Uniformly Sensitive Instrument for the Absolute Measurement of Sound Intensity," Physics Review, Vol. 10, No. 1, 1917, pp. 39-63. 